\title{
EchoGéo
}

$13 \mid 2010$

Afrique, 50 ans d'indépendance : État et territoires

\section{Les déterminants de la dynamique spatiale de la ville de Bingerville (sud de la Côte d'Ivoire) de 1960 à nos jours}

\section{Akou Don Franck Valéry Loba}

\section{CpenEdition}

Journals

Édition électronique

URL : https://journals.openedition.org/echogeo/12078

DOI : $10.4000 /$ echogeo.12078

ISSN : 1963-1197

Éditeur

Pôle de recherche pour l'organisation et la diffusion de l'information géographique (CNRS UMR 8586)

Référence électronique

Akou Don Franck Valéry Loba, "Les déterminants de la dynamique spatiale de la ville de Bingerville (sud de la Côte d'Ivoire) de 1960 à nos jours », EchoGéo [En ligne], 13 | 2010, mis en ligne le 20 septembre 2010, consulté le 21 septembre 2021. URL : http://journals.openedition.org/echogeo/ 12078 ; DOI : https://doi.org/10.4000/echogeo.12078

Ce document a été généré automatiquement le 21 septembre 2021.

EchoGéo est mis à disposition selon les termes de la licence Creative Commons Attribution - Pas d'Utilisation Commerciale - Pas de Modification 4.0 International (CC BY-NC-ND) 


\title{
Les déterminants de la dynamique spatiale de la ville de Bingerville (sud de la Côte d'Ivoire) de 1960 à nos jours
}

\author{
Akou Don Franck Valéry Loba
}

\section{Introduction}

Située à 18 kilomètres à l'est du centre ville d'Abidjan, Bingerville ${ }^{1}$ est une ville historique du sud de la Côte d'Ivoire. Sa création remonte à l'ère coloniale et repose en grande partie sur son statut de capitale, acquis en 1900. A la suite du transfert de la capitale sur le site d'Abidjan en $1934^{2}$, Bingerville devint un centre urbain ordinaire (Kipré, 1985). Après l'indépendance, la ville n'est plus qu'un chef-lieude souspréfecture. Du fait de son positionnement à la périphérie d'Abidjan, Bingerville bénéficie d'un statut particulier. La relative disponibilité en espaces urbanisables et l'existence d'un réseau routier facilitant son accès, sont les principales raisons qui ont fait d'elle une ville dortoir. En effet, Bingerville abrite de nombreux travailleurs abidjanais (Hauhouot, 1982 ; Koffi, 1987). Environ $1 / 5^{\mathrm{e}}$ des actifs résidents de Bingerville exercent une activité professionnelle à Abidjan (BNETD, 1993). Koffi (1987) indique que c'est à dessein que l'Etat a encouragé les promotions immobilières de la SICOGI, afin d'y fixer le maximum de travailleurs.Depuis 1975, la population de Bingerville n'a cessé de croître. Elle est passée de 18000 habitants à 35000 en 1998, soit un accroissement moyen annuel de 2,96 \% (Ministère du plan, 1988 ; Loba, 2008). On l'estime en 2010 à 50000 habitants.

2 Outre sa fonction de cité dortoir, Bingerville est un important centre scolaire. C'est d'ailleurs cette fonction héritée de son passé colonial qui s'est vue confirmer après l'indépendance. La ville abrite diverses institutions scolaires et des centres de 
formation professionnelle. A ce titre, ellea été le cadre de quelques investissements publics.

3 En dépit de ces atouts, Bingerville connait une faible urbanisation. Le paysage urbain se singularise par sa monotonie et sa vétusté. La dynamique spatiale est contrariée par la lenteur de la mise en valeur des terrains urbains. La ville est restée des années durant composée par les mêmes quartiers. Entre 1960 et 1980, la croissance des espaces bâtis y a été lente et peu significative, on est passé de 118 à 200 ha.ll faut attendrela décennie 1990-2000 pour voir de nouveaux lotissements donner lieu à de vastes chantiers à la périphérie du noyau initial. Depuis, l'espace bâti connaît un regain de croissance. De 400 ha en 1989, il est aujourd'hui estimé à plus de 1000 ha. Notre étude veut interroger les facteurs et les déterminants de cette croissance à deux vitesses. Quelles sont les causes de la stagnation entre 1960 et 1980 ? Et pourquoi ce brusque regain de croissance à partir de 1990 ?

4 L'objectif poursuivi est de produire une analyse en relation avec les grandes phases de l'histoire politique et économique nationale de 1960 à nos jours.

5 Nous avons utilisé la carte topographique de Bingerville issue de l'IGCI ${ }^{3}$ (feuille NB-30VIII-2bd-nb-30-IX-X-1ac, au 1/50 000). Des photographies aériennes ont aussi fourni les informations relatives aux mutations survenues dans l'occupation du sol. Elles proviennent du BNETD (Bureau National d'Etudes Techniques de Développement) et sont consécutives aux missions aériennes de 1965-1966, 1977, 1989-1990 et 1991, mais aussi des services du cadastre (2009). La réunion de toutes ces sources cartographiques a permis de réaliser une carte de synthèse relatant la dynamique spatiale de Bingerville (illustration 5). Ces sources ont aussi contribué à calculer l'aire du bâti aux dates cidessus mentionnées.

A partir des recensements de l'institut national de la statistique (INS) de 1975, 1998 et 1998, et des estimations de 2008, nous avons pu obtenir les effectifs de la population de Bingerville et mesurer leur dynamique.

6 Auprès du service technique municipal, nous avons obtenu des informations relatives aux équipements et à la gestion du domaine foncier. Les registres consultés auprès du secrétariat général et du service financier nous ont renseigné sur la planification des projets de développement et sur la mobilisation des ressources financières émanant de la gestion du foncier. Ces informations ont permis de montrer la dynamique des paysages urbains en terme de gestion environnementale et de dotation en équipements (Yapi-Dihaou, 2003). 
Illustration 1 - Bingerville dans le District d'Abidjan

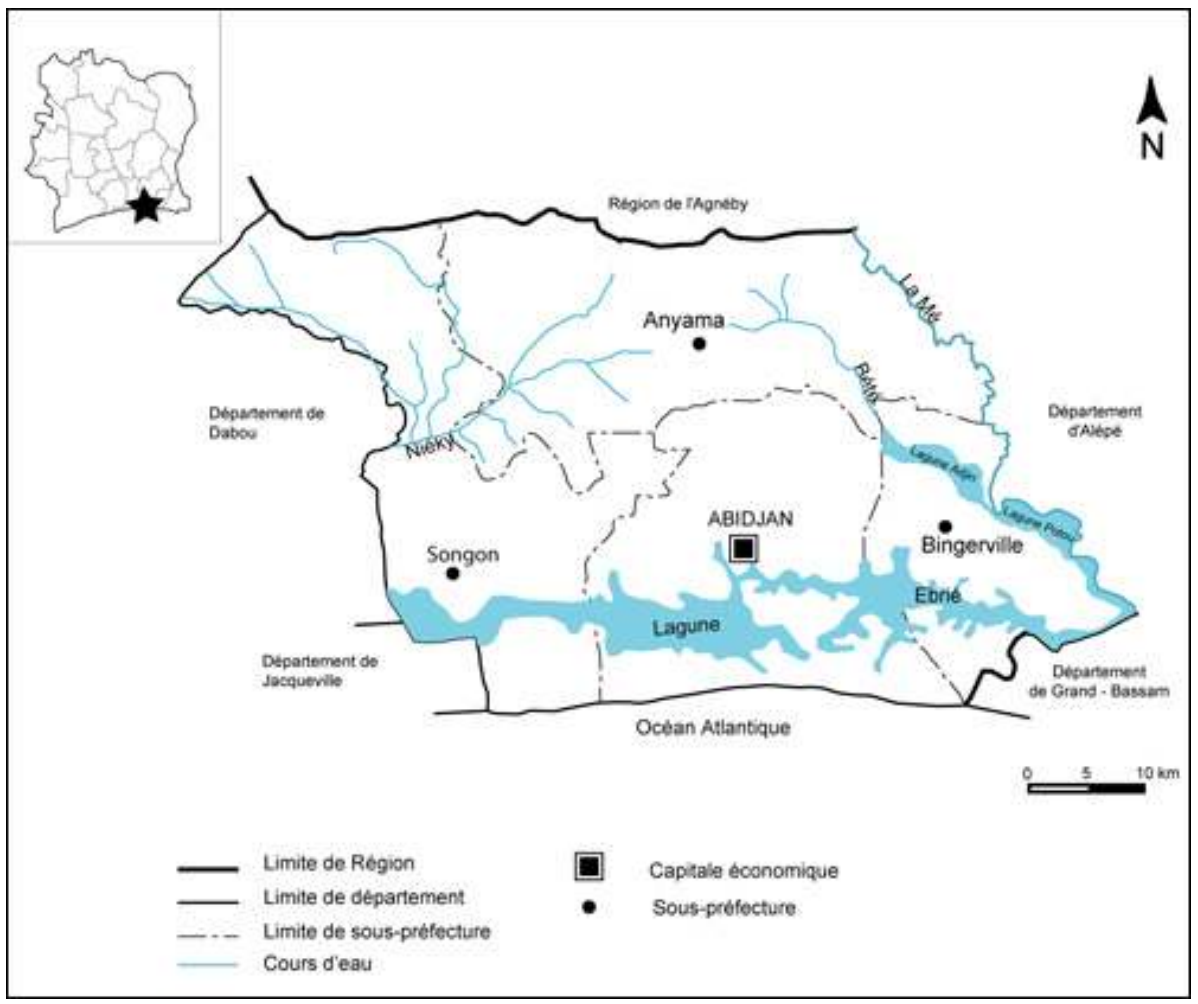

Source : ministère de l'intérieur et de la décentralisation, 2009.

\section{Construction urbaine et "protectionnisme Ebrié » ${ }^{4}$}

\section{Le soutien des structures de l'Etat au processus d'urbanisation}

7 L'Etat de Côte d'Ivoire après son accession à l'indépendance a soutenu l'urbanisation par l'entremise de structures spécialisées comme la SICOGI (Société Ivoirienne de Construction et de Gestion Immobilière). Les retombées du négoce international ont permis le financement de l'ambitieux programme de développement urbain initié par l'Etat depuis le plan quinquennal de 1961-1965 (Atta, 1975, 2000). Les opérations de la SICOGI ont été rendues possible par les ressources issues de la vente de matières premières telles que le café et le cacao. On parlait à cette époque du «miracle économique ivoirien ». A Bingerville, les interventions de la SICOGI ont permis la construction de logements de moyen et de haut standing. Ces habitations étaient destinées pour la plupart aux familles de fonctionnaires issus de diverses structures publiques et parapubliques (Koffi, 1987). Cette initiative de l'Etat correspondait à la volonté des pouvoirs publics de désengorger Abidjan.

Malheureusement, cette volonté des pouvoirs publics sera contrariée par laméfiance des populations autochtones, décidées à ne pas laisser leur territoire aux mains « d'étrangers » (Pony, 1992 ; Terrier, 1995 ; Loba, 2009), et qui redoutent deperdre le contrôle de leurs terres. Les Ebrié contestaient donc l'affirmation du droit régalien de l'Etat et exigeaient des indemnisations. Soutenus par leur chefferie, ils se montrèrent hostiles au projet de l'Etat, allant même jusqu'à intimider l'administration domaniale. Cette attitude a constitué une entrave à la dynamique spatiale de la ville (Koffi, 1987 ; 
BNETD, 1993). Ainsi, la confrontation entre droit coutumier et droit moderne constitue l'un des problèmes majeur de la mise en valeur des domaines urbains. L'urbanisation de la ville amorcée par la SICOGI s'en est trouvée fragilisée (Kobo, 1985).

\section{Illustration 2 - La résidence Coulibaly à Bingerville}

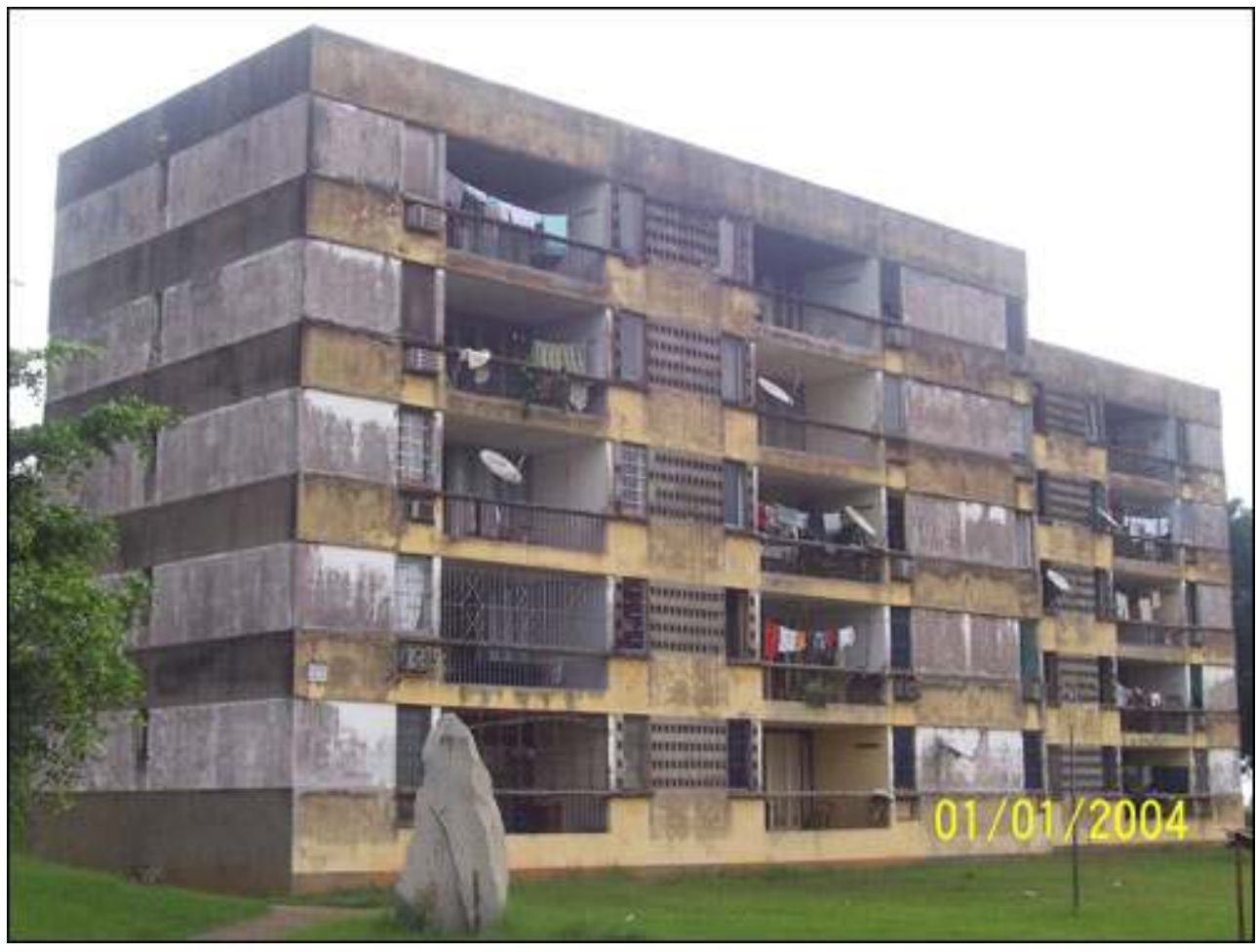

Des appartements en hauteur bâtis en 1975 par la SICOGI et financés exclusivement par l'Etat.

Auteur : Loba Akou, 2004.

9 La mise en valeur du foncier à Bingerville fut donc problématique. En effet, du fait de la proximité de la banlieue de la métropole abidjanaise, que Bingerville intégrait progressivement, les autochtones craignaient de se voir phagocyter. Déjà refoulés de leurs terres par le colonisateur lors de la construction de la ville en 1900, et s'inspirant des situations vécues par les Ebrié d'Abidjan, dont l'espace culturel (notamment à Cocody, Koumassi, et Adjamé) ${ }^{5}$ a été absorbé par la fulgurante croissance d'Abidjan, les Ebrié de Bingerville ont pendant longtemps refusé de rétrocéder leurs terroirs (Pony, 1992 ; BNETD, 1997).

10 Ainsi, au cours de la décennie 1970, faute de terrains disponibles, l'administration domaniale n'a pu satisfaire les nombreux demandeurs de lots voulant exploiter le front lagunaire.Entre 1975 et 1980, la direction de l'urbanisme et de la ZAD (Zone d'Aménagement Différé) constata que $80 \%$ des demandeurs étaient des travailleurs abidjanais. Ces derniers étaient essentiellement motivés par le coût relativement bas des logements, par opposition à Abidjan, où en plus du prix, l'accès au foncier n'était pas facile. Pourtant, à Bingerville aussi, il fut difficile de satisfaire les candidats. L'administrateur civil en poste au début des années 1980 est resté impuissant (Koffi, 1987 ; Koffi, 2004).

11 La léthargie que vécut la ville jusqu'en 1985 s'explique en partie par cette confrontation entre les populations autochtones et le gouvernement. En dépit du contexte 
économique national favorable, la puissance publique n'a pu, en dehors des opérations immobilières limitées de la SICOGI, amplifier l'urbanisation de la ville, faute de terrains disponibles.

\section{Ruines coloniales et dualité sociospatiale}

Bingerville se particularise par la présence d'un patrimoine architectural hérité de l'époque coloniale. Sur le plateau central de la ville, au cœur du quartier résidentiel, le paysage urbain est dominé par d'imposantes bâtisses datant de l'ère coloniale et dont la plus remarquable est l'ancien palais du gouverneur (illustration 3). Cette bâtisse surplombant la ville, accueille depuis l'indépendance l'orphelinat national. Un peu plus au sud, l'ancien bâtiment du service des postes colonial abrite la sous-préfecture. Ces monuments témoignent du passé glorieux de Bingerville. Malheureusement, ils ne sont pas convenablement entretenus et tombent progressivement en ruine. Ce quartier ne fait pas patrimoine pour les autochtones Ebrié qui n'y vivaient pas. Il n'est pas assez important pour susciter l'intérêt de l'Etat qui s'est jusqu'à présent prioritairement intéressé au patrimoine colonial de Grand Bassam, dont le classement au patrimoine mondial de l'Unesco est actuellement à l'étude.

Illustration 3 - Le palais du gouverneur de la colonie

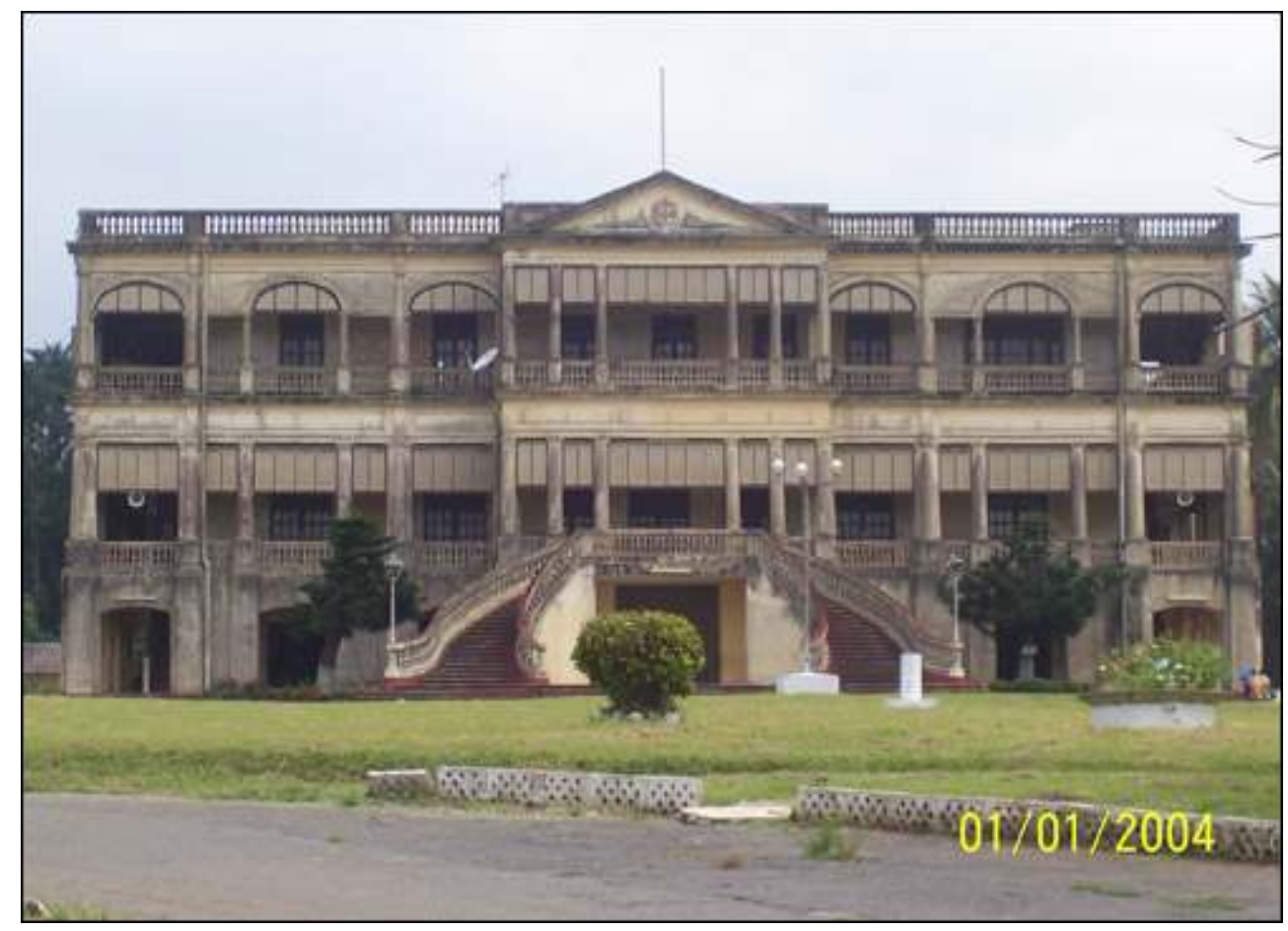

La présence de cet édifice singularise le paysage urbain de Bingerville.

Auteur : Loba Akou, 2004

Au-delà du modeste centre colonial, le tissu urbain est caractérisé par une franche dualité sociospatiale. En effet, s'opposant à l'application du droit foncier moderne, les populations autochtones lotirent elles-mêmes leurs terres et procédèrent à des attributions familiales (Pony, 1992) qui furent ensuite l'objet de locations et de ventes. Les locations de terrains, mode le plus répandu, répondaient au besoin de ne pas voir les terres échapper à leur contrôle. Les locataires étaient, pour la plupart, des 
ressortissants de la CEDEAO ${ }^{6}$. Ces populations en quête d'emplois dans le secteur formel exercent en attendant de petites activités informelles leur permettant de subvenir à leurs besoins quotidiens. Ce sont « les exclus de la ville » (Casez et Domingo, 1991). Leur profil socio-économique a favorisé l'occupation anarchique de l'espace. Il s'en est suivi un développement de l'habitat précaire au sud de la ville, à Gbagba, où l'essentiel de l'habitat échappe aux normes urbanistiques. On y a observé la prolifération d'habitations de type individuel bâties avec toutes sortes de matériaux.

Gbagba est l'un des plus vieux quartiers de la ville. Pendant l'occupation française, il était essentiellement peuplé d'indigènes. Gbagba est aujourd'hui composé d'une extension et d'un impressionnant sous-quartier dénommé "Sans-loi ». Ce vaste ensemble situé en contrebas d'une pente abrupte regroupe desformes d'habitats non conventionnels et qui contrastent avec le reste de la ville. Il se crée dans le paysage urbain une dualité visible avec d'un côté la ville formelle composée par les résidences de la SICOGI et de l'autre les terrains mis en location par les autochtones, où essaiment des baraquements. Les nombreux conflits fonciers ont favorisé une urbanisation parallèle donnant par endroit à la ville l'aspect d'un village.

\section{La Communalisation au secours du développement urbain}

\section{L'apparition de nouveaux acteurs sur le marché du foncier}

Le début des années 1980 est marqué par un changement institutionnel majeur. Cette période se caractérise par la relance de la politique de décentralisation, sur recommandation des institutions financières internationales (colloque international, 1988; Yapi-Dihaou, 1990; Gentil et al, 2004). Ce changement intervient dans un environnement économique national marqué par la récession et les programmes d'ajustement structurel (Stren et White, 1993 ; Le Bris, 1998). Ainsi, l'Etat se voit obligé de surseoir aux investissements dans les secteurs sociaux, dont l'immobilier. Des opérateurs privés, en partenariat avec les nouveaux pouvoirs locaux (communes), vont à cet effet le relayer (Bertrand, 2002 ; Loba, 2008).

16 La commune se positionne depuis lors comme le véritable animateur du marché foncier, et ce, en dépit des conflits de compétences avec la sous-préfecture. Depuis la dévaluation du franc CFA en 1994 et le début des années 2000, la présence des structures immobilières privées s'est accrue (voir illustration 5). De nombreuses sociétés immobilières s'intéressent aux terroirs de Bingerville. Les opérateurs ${ }^{7}$ les plus importants sont PROMOGIM (Société de promotion et de gestion immobilière), les Figuiers, le BNETD et le Trésor public. Progressivement, de nouveaux quartiers ont contribué à rapprocher la ville de Bingerville de la partie orientale de l'agglomération abidjanaise. Cette situation est nettement illustrée par les nouvelles opérations immobilières réalisées sur les marges septentrionales (N'gotto, N'gbromin) et occidentales (lotissements privés et villageois) de Bingerville. 
Illustration 4 - Evolution des espaces urbanisés de la ville de Bingerville

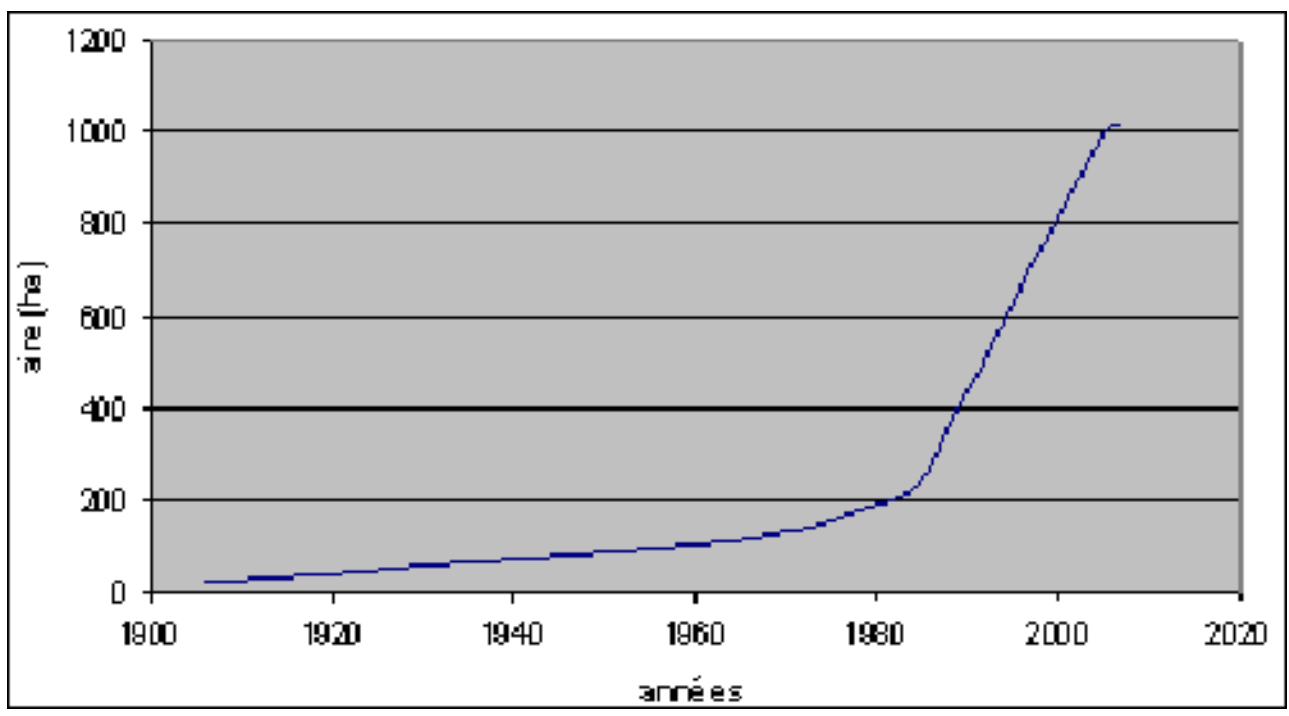

Source : BNETD-CCT 1965, 1977, 1989 et 1991 ; Cadastre, 2009.

17 Ces récentes extensions du tissu urbain sont les lieux où émerge une nouvelle forme de paysage caractérisé par un habitat de moyen et de haut standing. Progressivement, ces quartiers se peuplent. Leurs occupants sont majoritairement des travailleurs abidjanais (Koffi, 2004 ; Loba, 2008). La densité y est encore faible mais elle augmente rapidement. Les nouveaux lotissements se présentent dans le tissu urbain comme des greffes juxtaposées au noyau initial. La ville compte désormais plus d'une dizaine de quartiers, présentant des paysages très variés. Sa superficie bâtie est évaluée à plus de 1000 ha (Loba, 2008) contre 118 en 1966. Les terrains des nouvelles opérations immobilières sont viabilisés et disposent de toutes les commodités (voirie, adduction en eau potable, raccordement au réseau électrique et de téléphonie, réseau d'assainissement). En dépit de cette évolution, Bingerville n'a pas un centre ville bien délimité. Cette situation est un héritage du passé colonial de la ville. En effet, l'administration coloniale avait, suivant les règles ségrégatives de l'époque, opéré une nette séparation entre les quartiers suivant leur fonction (administration, commerce, résidence des Européens, des Africains). A cette fragmentation s'ajoute le morcellement du site par de nombreux thalwegs, qui accentue la dualité du paysage urbain: d'un côté, la ville légale, sur l'interfluve, et de l'autre, en contrebas, la ville spontanée, symbolisée par le bidonville de Gbagba. 


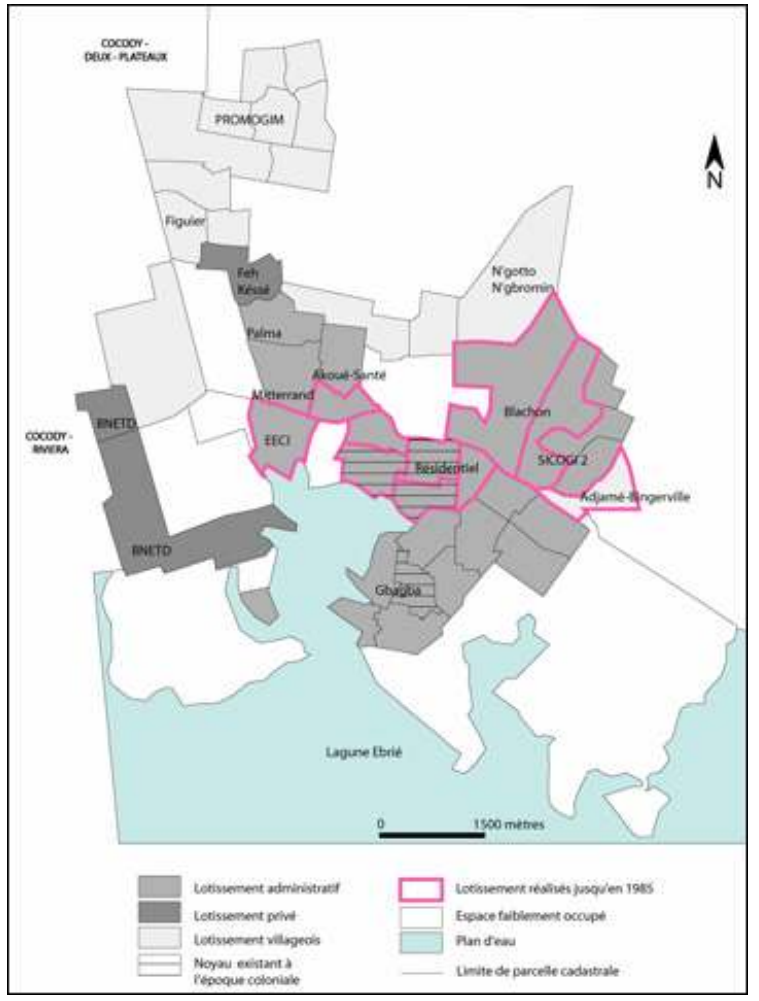

Source : direction du cadatre, 2009.

\section{La commune et les nouveaux mécanismes institutionnels de production du foncier urbain}

La mise en application de l'approche participative prévue par les textes législatifs a contribué à restaurer la confiance entre les élus locaux et les populations autochtones. Désormais, l'Etat, par le biais de la commune, est dans l'obligation de composer avec les détenteurs du droit coutumier. Quant aux chefs terriens, ils ont le devoir de soumettre un avis au maire pour approbation du lotissement. Une fois la municipalité avisée, la communauté villageoise peut saisir un géomètre et procéder à un état des lieux. Celuici est approuvé par la mairie puis par le ministère de la construction, après vérification par les géomètres de la Direction de l'urbanisme. Puis un plan parcellaire est préparé ainsi qu'un arrêté d'ouverture d'enquête d'incommodo. Cet arrêté revient au maire qui, en tant que président de la commission habilitée à diriger l'enquête, informe les autres membres de la commission.

Les textes adoptés depuis le retour au multipartisme en $1990^{8}$ ont pour objectif de freiner les ventes anarchiques de terrain, commedans la périphérie de Bingerville. La législation stipule désormais que seule l'immatriculation du titre foncier confère un droit définitif et inattaquable à la propriété.

Les mairies deviennent les initiatrices de la dynamique spatiale des villes, à travers l'exécution des projets de lotissements. En effet, le cadre législatif de la décentralisation, par le biais de la loi n ${ }^{\circ} 80-1180$ du 17 octobre 1980 (en son article 60 bis nouveau), impute aux communes la responsabilité du suivi de la collecte des recettes et particulièrement des impôts, taxes et droits municipaux. Pour la majorité 
des communes, les lotissements se sont révélés d'importantes ressources. Observons que le montant des recettes liées aux impôts fonciers a pratiquement été multiplié par 7 entre 2000 et 2002, où l'on est passé de près de 6,3 millions à plus de 49 pour Bingerville. Toujours sur la même période, selon les statistiques du service financier de la commune, les impôts fonciers ont rapporté beaucoup plus au budget que le petit commerce, qui demeure la principale activité économique. De ce fait, la mairie a tout intérêt à multiplier les projets de lotissement.

21 Les lotissements de N'gotto et de N'gbromin (nord de la ville sont le symbole de l'extension du périmètre urbain, dans un contexte socio-politique difficile marqué par la crise militaro-politique de septembre 2002 (Loba, 2008).

C'est principalement la loi $\mathrm{n}^{\circ}$ 85-582 du 29 juillet 1985 qui rend opérationnel ce transfert des compétences, qui concerne aussi les charges des équipements urbains et la mise en valeur du patrimoine foncier ${ }^{9}$. Cette tâche est donc confiée aux communes par le biais de l'élaboration des documents d'urbanisme et de la délivrance des autorisations d'utilisation des sols. On note qu'obligation leur est faite d'introduire une demande d'établissement de projets de lotissements lorsque le terrain est propriété de l'Etat. L'exercice de cette compétence contribue de façon significative à la dynamique spatiale. On observe ainsi que, depuis 1985, date de la communalisation, la municipalité a supplanté l'Etat dans le processus de création du sol urbain. Selon les services techniques de la mairie, plus des deux tiers de la production de terrain depuis 2002 émanent de la mairie et de la collaboration entre la mairie et les différents comités villageois. Dans cette nouvelle configuration, en attendant un transfert plus large des compétences tant souhaité par les maires, le sous-préfet intervient encore dans quelques cas dans l'attribution de certains lots. Cette situation a été très souvent à l'origine de conflits de compétence entravant le fonctionnement de la commission d'attribution des lots.

Ainsi, la communalisation a positionné la municipalité comme un nouvel et incontournable acteur de la production des terrains urbains après l'Etat et les autochtones Ebriés, encore considérés comme maitres de la terre du fait de leur statut de premier occupant.

\section{Et Bingerville redevient une périphérie attractive}

Depuis 2001, la nouvelle municipalité a entrepris de convaincre les chefs terriens du bien fondé de nouveaux lotissements. En retour, elle s'est engagée à les dédommager de façon conséquente, en leur octroyant des lots sur les terrains viabilisés. On enregistre depuis lors la délivrance de nombreux permis de construire attribués aux personnes non résidentes à Bingerville. Le nombre de permis de construire concédéaux non résidents a été multiplié par 4 entre 2001 et 2004. La proximité de la ville d'Abidjan en est, selon le service technique de la mairie, le principal facteur explicatif. Cela s'explique aussi par le fait qu'à Bingerville, les coûts d'acquisition de terrain sont relativement bas. Le prix du mètre carré du terrain nu est de 3000 francs CFA dans les quartiers résidentiels et de 2000 dans les quartiers d'habitat de type évolutif, très en deçà de ceux pratiqués à Abidjan (Loba, 2002; 2008). Du fait de sa proximitéé, Bingerville risque de connaître une trop forte demande, qui pourrait provoquer une hausse des prix, en particulier dans les quartiers résidentiels, où la demande est la plus importante. 
La ruée sur les terrains urbains à la périphérie du noyau initial de Bingerville se présente comme une tendance forte (Koffi, 2004; Loba 2008). L'amélioration des liaisons entre les deux villes par l'ouverture de deux lignes express par la SOTRA (Société de Transport Abidjanais) accroît les mouvements pendulaires.

Pour la municipalité, ces avancées constituent de véritables réussites. Du fait de problèmes multiples dans les sphères familiales liés à la succession ou au partage de terrain, Bingerville stagnait en matière de production foncière, ce qui freinait son expansion et la viabilisation des parcelles à la périphérie de la ville. En effet, à la suite des distributions anarchiques de parcelles et de leur mise en valeur, nombreuses sont les familles dont les membres étaient entrés en conflit. Refusant l'arbitrage des autorités, chacun évoluait à sa guise, échappant à tout contrôle. C'est à cette situation dommageableque la municipalité a pu apporter un début de solution.

Illustration 6 - Evolution des attributions de permis de construire dans la commune de Bingerville de 1987 à 2006

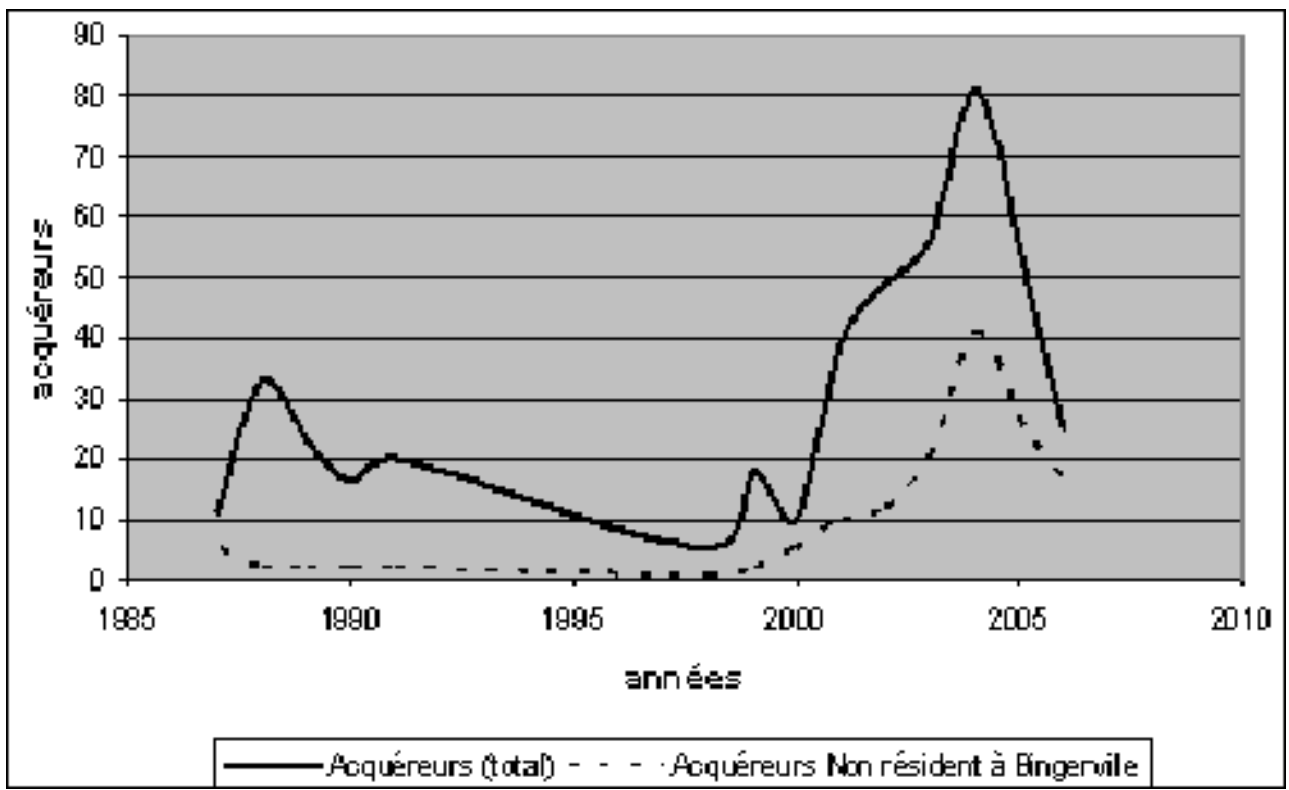

Le graphique indique une nette évolution des attributions de terrain à partir de l'an 2000. Cette croissance est le fait de l'amélioration des relations entre les autochtones et la municipalité. La baisse observée après 2005 s'explique par la situation de crise que connaît la Côte d'Ivoire.

Source : services techniques mairie de Bingerville, 2007. 
Illustration 7 - Promotion immobilière de la cité "Feh Kessé "; symbole de l'extension de Bingerville vers l'ouest à la limite de la commune résidentielle de Cocody (à l'est d'Abidjan)

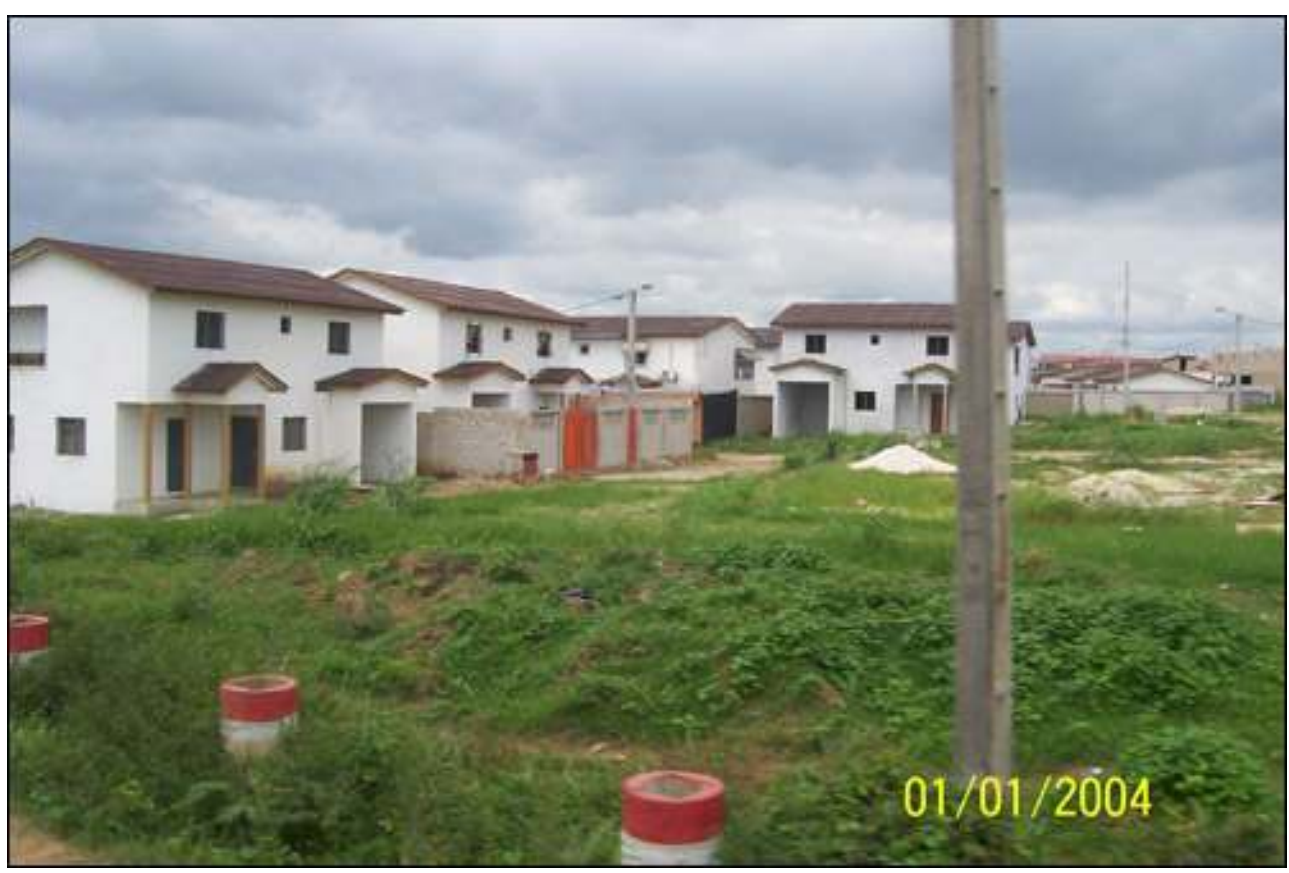

Auteur : Loba Akou, 2004

\section{Conclusion}

Les relations conflictuelles entre populations autochtones et pouvoirs publics et la politique de communalisation sont les principaux déterminants de la dynamique spatiale de Bingerville. Si les oppositions entre droit coutumier et droit régalien ont freiné depuis 1970 la croissance de la ville, la décentralisation de 1985 a progressivement inversé la tendance. Les nouvelles procédures administratives foncières introduites par la communalisation sont parvenues à réguler la production de terrains urbains.

Ainsi, autour du noyau colonial, de nouveaux quartiers ont été édifiés. La configuration de la ville de Bingerville a considérablement évolué. En très peu de temps, Bingerville s'est brusquement rapprochée d'Abidjan. La limite entre les deux villes est devenue difficilement perceptible. La coalescence des fronts d'urbanisation des deux villes est à présent source de maints litiges fonciers entre les communes de Bingerville et de Cocody. La fulgurante croissance des périphéries urbaines met à l'ordre du jour l'épineuse question de la délimitation des périmètres communaux et de la gestion du foncier. Jusqu'à ce jour les autorités en charge du dossier ont éludé cet épineux problème. Sa persistance mérite une attention particulière de la part des chercheurs et des pouvoirs locaux. 


\section{BIBLIOGRAPHIE}

Antoine P., Dubresson A., Manou-Savina A., 1987. Abidjan « côté cours » : pour comprendre la question de l'habitat. Paris, ORSTOM, Karthala, $277 \mathrm{p}$

Atta K., 1975. Etude des espaces urbains des villes de Côte d'Ivoire. Université de Côte d'Ivoire, Abidjan, Mémoire Maîtrise, IGT, 120 p.

Atta K., 2000. Urbanisation et développement en Côte d'Ivoire. Université de Côte d'Ivoire, Abidjan, IGT ,65 p.

Bertrand M., 2002, Gestion foncière et logique de projet urbain : expériences comparées en Afrique occidentale, francophone et anglophone. In Regards sur l'Afrique, La Renaissance de la Géographie à l'Aube du Troisième Millénaire : Conférence Régionale de l'UGI, 2002/08, Durban. Historiens et Géographes, (379), p. 77-89.

Bertoncello B., Bredeloup S., 2002. La privatisation des marchés urbains à Abidjan : une affaire en or pour quelques-uns seulement. In Gérer la ville : entre global et local. Paris, Autrepart, 21, p. 83-100.

BNETD, 1993. Projet de développement des communes côtières; communes de Bingerville. 55 p.

BNETD, 1997. P.D.C.C 6e et 7e FED étude technique et financière ville de Bingerville. 1ère phase, avenant

Cazes G., Domingo J., 1991. Le sous développement et ses critères. Paris, éd. Boréal, 255 p.

Choplin A., 2006. Le foncier urbain en Afrique : entre informel et rationnel, l'exemple de Nouakchott, capitale de la Mauritanie. Annales de Géographie, n 647, Armand Colin, p. 69-91.

Colloque international, 1988. La décentralisation : études comparées des législations ivoiriennes et françaises. Centre de valorisation de la recherche de Toulouse, $228 \mathrm{p}$.

Dubresson A., Faure Y-A., Freund B., Lootvoet B., Piveteau A., Estrada M., Labazee P., Kennedy L., 2005, Décentralisation et développement local : un lien à repenser. Paris, Revue Tiers Monde, 46 (181), $241 \mathrm{p}$.

Gentil D. et al, 2004. La décentralisation en Afrique de l'ouest entre politique et développement. Paris, 408 p.

Hauhouot A., 1982. Dynamique de l'espace ethno villageois d'Abidjan. Annales de l'université d'Abidjan série G, tome XI. Géographie, p. ${ }^{\circ 9}$-25.

Kobo P., 1985. L'urbanisation et les droits coutumiers. Abidjan, cités africaines (2), p. 28-31.

Koffi A., 1987. Etude comparée de la dynamique de l'espace urbain à Bingerville et à Anyama. Abidjan, Université de Cocody, mémoire de maîtrise, IGT, $117 \mathrm{p}$.

Koffi Y., 2004. Gestion du foncier urbain : cas de Bingerville. Bouaké, Université de Bouaké, mémoire de maîtrise, UFR-CMS, $114 \mathrm{p}$.

Konan B., 1998. Les dix chantiers du futur, bilan du programme présidentiel à mi parcours. Abidjan, Documentation Ivoirienne, $200 \mathrm{p}$.

Kipre P., 1985. Villes de Côte d'Ivoire 1893-1940. Abidjan, NEI, Tome I, 238 p. ; tome II, 290 p. 
Le Bris E., 1998. Urbanisation et politiques urbaines dans les pays en développement, in Populations et développements : une approche globale et systémique. Paris : Academia-Bruylant, L'Harmattan, p. 297-356.

Loba A., 2002. Inventaire et cartographie dynamique des équipements et des infrastructures dans les départements d'Abidjan et d'Alépé. Abidjan, Université de Cocody, mémoire de maîtrise, IGT, 103 p.

Loba A., 2008. Dynamique de développement des villes côtières dans la région des lagunes : cas de Bingerville, Dabou et Grand-Lahou. Abidjan, Université de Cocody, Thèse unique, IGT, 389 p.

Loba A., 2009. Impact de la progression du front d'urbanisation abidjanais sur le developpment de la communauté villageoise de M'badon (village Ebrié). Revue du LADRYMES, université de Lomé, p. 79-92.

Martinaud C., Marchand Y., 1996. Les défis de l'urbanisation. La documentation française, Paris, p. 12-19.

Marques-Perreira J., 1987. Les enjeux politiques de la question urbaine au Brésil : citoyenneté, conflits urbains et identités populaires. Paris, cahiers des sciences humaines, (23) 1, p. 131-147.

Ministère du Plan, 1988. RGPH résultats provisoire. Abidjan, 4é éd., $187 \mathrm{p}$.

Haeringer P., 1970. La croissance urbaine en Afrique noire, cas de San-Pedro. CNRS, Paris, p. 625-651.

PDM, 2003. Etat de la décentralisation en Afrique. Paris, Khatarla, $230 \mathrm{p}$.

PDM, 2006. Africités, session thématique : comment vont être financé les besoins en investissement des villes africaines. Rapport de synthèse, Nairobi, 5 p.

Pony L., 1992. Le problème foncier Ebrié face au peuplement d'Abidjan. Paris, Université Paris 1 Sorbonne, mémoire de DEA, 55p.

Stren R., White R., 1993. Villes africaines en crise. Paris, L'harmattan, 345 p.

Terrier C., 1995. De la terre au territoire: Récit du parcours Ebrié ou gens de la terre au pouvoir dans la société Abidjanaise (Côte d'ivoire). Paris, ORSTOM, 9 p.

Yapi-Dihaou A., 1988. Politique urbaine en Côte d'Ivoire, son impact sur l'évolution des formes d'habitat non planifié : l'exemple de l'agglomération abidjanaise . Abidjan, ORSTOM, $37 \mathrm{p}$.

Yapi-Dihaou A., 1990. L'Etat et les municipalités en Côte d'Ivoire : un jeu de cache-cache? In Le droit et ses pratiques, Politique Africaine, 1990, (40), p. 51-59.

Yapi-Diahou A., 2003. La recherche urbaine à l'épreuve des milieux marginalisés dans la ville. Abidjan, EDUCI, $123 \mathrm{p}$.

\section{NOTES}

1. Alobey est le nom originel de la ville. C'est en hommage à Louis Gustave Binger (1856-1936) explorateur puis gouverneur de la Côte d'Ivoire française, que la localité fut baptisée Bingerville.

2. L'évacuation des matières premières vers la métropole nécessitait la création d'un port en eau profonde qui serait en même temps le terminus d'une liaison ferroviaire desservant l'hinterland. C'est ainsi que sous le commandement du capitaine Houdaille, la puissance coloniale entreprit la recherche d'un site pouvant abriter l'infrastructure portuaire. Le site de Santey (Abidjan) s'est présenté aux yeux des membres de l'expédition comme le lieu le plus approprié (Kipré, 1985).

3. Institut Géographique de Côte d'Ivoire.

4. Population autochtone de la région de Bingerville. 
5. Ce sont des communes du district d'Abidjan, constituées à partir de villages Ebrié. Dans ces communes, la situation est différente de ce qui prévaut à Bingerville. Le droit régalien de l'Etat a pu s'imposer depuis l'époque coloniale. D’importantes étendues ont été de facto réquisitionnées pour la construction de la capitale économique (Kobo, 1985 ; Loba, 2009).

6. Communauté Economique des Etats de l'Afrique de l'Ouest.

7. Les constructions immobilières occupant la périphérie de Bingerville sont majoritairement le fait d'opérateurs privés installés en Côte d'Ivoire après la dévaluation du franc CFA en 1994. Les capitaux de ces sociétés sont détenus par des bailleurs européens, en l'occurrence français (Konan, 1998).

8. Les dispositions contenues dans le décret $n^{\circ}$ 77-906 du 6 novembre 1977 relatif aux lotissements villageois et le décret $\mathrm{n}^{\circ}$ 92-398 du $1^{\mathrm{er}}$ juillet 1992 portant réglementation du permis de construire montrent la volonté de l'Etat d'associer les chefs coutumiers à la gestion du foncier. 9. Le patrimoine foncier en pays Ebrié se compose de l'ensemble des terres acquises par les grandes familles. La gestion de ce patrimoine est communautaire (Bertrand., 2002 ; Bertoncello et Bredeloup., 2002).

10. Par voie routière, en l'absence d'embouteillage, Bingerville est à moins de 15 minutes du centre d'Abidjan (BNETD,1993).

\section{RÉSUMÉS}

Le présent article rend compte des déterminants de la dynamique spatiale de Bingerville, une ville historique du sud de la Côte d'Ivoire. Les sources cartographiques émanant de missions aériennes ont permis de faire la synthèse de la dynamique spatiale. Une enquête auprès du service technique de la mairie a fourni les informations relatives à la gestion du foncier.

L'étude révèle que de 1960, date de proclamation de l'indépendance, jusqu'en 1985, en dépit des interventions de l'Etat-providence, la croissance de la tache urbaine a été très peu significative. A la volonté de l'Etat de bâtir une ville moderne se sont opposées la méfiance et l'hostilité des autochtones, redoutant d'éventuelles expropriations.

Avec les changements institutionnels survenus après 1985, qui ont vu l'apparition de nouveaux pouvoirs locaux décentralisés, la ville va connaître un regain de croissance. La communalisation, en mettant à l'ordre du jour l'implication des populations autochtones dans les mécanismes de création du sol urbain, est parvenue à relancer la dynamique des lotissements. Ainsi, sous l'impulsion d'opérateurs immobiliers privés, la ville a vu s'ajouter à son noyau initial de nouveaux quartiers ; elle connaît depuis lors un étirement en direction de ses périphéries ouest et nord et devient partie intégrante du front d'urbanisation oriental de la ville d'Abidjan.

This article reports on the spatial dynamics of Bingerville, a historic city in southern Côte d'Ivoire. The study aims to examine data on the nature of the determinants of this dynamic space. The cartographic sources from aerial missions were allowed to synthesize spatial dynamics. A survey of the technical department of the council has provided information on land management.In 1960, the date of declaration of independence until 1985, despite the interventions of the welfare state, the growth of the urban stain was very insignificant. A commitment to the state to build a modern city, opposed the distrust and hostility of the indigenous, fearing possible expropriations.

During the period of economic recession, marked by the advent of structural adjustment 
programs, the process of urbanization Bingerville will experience a revival. The institutional changes that occurred after 1985 which saw the emergence of new local authorities in this case the county, there are many. The communalisation, building on the agenda involve the population in autocthtones mechanisms of creation of urban land, managed to regain the momentum of developments. Thus, under the impetus of private real estate operators, the city has seen in addition to its original core of new neighborhoods, it has since met with stretching toward its northern and western suburbs and become part of the front of urbanization East of the city of Abidjan.

\section{INDEX}

Mots-clés : Bingerville, communalisation, Dynamique spatiale, Ebrié, foncier, lotissement Keywords : Bingerville, Ebrié, land, spatial dynamics, subdivision communalisation

\section{AUTEUR}

\section{AKOU DON FRANCK VALÉRY LOBA}

Akou Don Franck Valéry Loba est Enseignant-chercheur à l'Institut de Géographie Tropicale de l’Université de Cocody - Abidjan. valo226@yahoo.com 